Revista peruana de biología 26(4): 475 - 480 (2019) doi: http://dx.doi.org/10.15381/rpb.v26i4.17216 ISSN-L 1561-0837; eISSN: 1727-9933

Universidad Nacional Mayor de San Marcos

\section{Evaluación de la abundancia relativa de Telmatobius culeus en la zona litoral del lago Titicaca, Perú}

\section{TRABAJOS ORIGINALES}

$\begin{array}{ll}\text { Presentado: } & 11 / 06 / 2019 \\ \text { Aceptado: } & 26 / 11 / 2019 \\ \text { Publicado online: } & 16 / 12 / 2019\end{array}$

\section{Correspondencia:}

Victor E. Ramos Rodrigo: ver_viento@hotmail.com Jhazel A. Quispe Coila: jhazelqc@gmail.com Roberto K. Elias Piperis: roberto.elias@upch.pe

2 Av. Honorio Delgado 430, San Martin de Porres, Lima, Perú.

\section{Assessment of relative abundance of Telmatobius culeus in littoral zone of Lake Titicaca, Peru}

\section{Víctor Enrique Ramos Rodrigo*1, Jhazel Arnold Quispe Coila ${ }^{3}$ y Roberto Kosmas Elias Piperis ${ }^{1,2}$}

1 Denver Zoological Foundation, USA.

2 Universidad Peruana Cayetano Heredia, Lima, Perú.

3 Asociación para la Ciencia y el Desarrollo Ambiental, Natural Way, Puno, Perú.
Otros datos de los autores / biografía:

ORCID Victor E. Ramos: 0000-0002-1531-1835 ORCID Jhazel A. Quispe: 0000-0001-6798-9528 ORCID Roberto K. Elias: 0000-0003-4271-4354

Citación:

Ramos Rodrigo V.E., J.A. Quispe Coila y R. K. Elias Piperis. 2019. Evaluación de la abundancia relativa de Telmatobius culeus en la zona litoral del lago Titicaca, Perú. Revista peruana de biología 26(4): 475 - 480 (Diciembre 2019). doi: http://dx.doi. org/10.15381/rpb.v26i4.17216

Palabras clave: Telmatobius; Rana Gigante del Titicaca; lago Titicaca; abundancia; hábitat; Andes; Puno; endémico.

Keywords: Telmatobius; Titicaca Water Frog, Lake Titicaca; abundance; habitat; Andes; Puno; endemic.

\section{Resumen}

El estudio se realizó en 13 zonas del litoral peruano del lago Titicaca, durante la época seca, desde junio hasta agosto de 2017. En cada zona se determinó la abundancia relativa y uso de microhábitat de Telmatobius culeus. En las zonas de evaluación se utilizó buceo con esnórquel para realizar en cada una tres transectos de 100 $\mathrm{m} \times 2 \mathrm{~m}$, con un total de 38 transectos evaluados. Se registraron 45 individuos en solo seis zonas de evaluación. El mayor número de individuos se encontraron en la zona de Perka Norte, con una abundancia relativa de 10.33 individuos/hora/02 personas a una profundidad de $1.5 \mathrm{~m}$; en siete zonas no se registró ningún individuo. Los individuos fueron encontrados en los siguientes micro hábitats: 17 fueron encontrados sobre la vegetación acuática, 11 en arena combinado con vegetación acuática, 9 en roca combinado con vegetación acuática, 5 en fango combinado con vegetación acuática, 2 en roca y 1 en arena. a la vez se realizó análisis de diferentes parámetros de calidad de agua en cada zona de estudio, como: temperatura, $\mathrm{pH}$, alcalinidad, dureza y oxígeno.

\section{Abstract}

The Telmatobius culeus relative abundance and its microhabitat use was determined. In 13 areas of Peruvian littoral of Lake Titicaca was carried out, during the dry season, since June to August 2017. In each the evaluation areas, snorkeling was used to perform three $100 \times 2 \mathrm{~m}$ transects. A total of 38 transects were evaluated. 45 individuals were registered in only six evaluation zones. The largest number of individuals were found in the North Perka area, with a relative abundance of 10.33 individuals/hour/02 people, at a depth of $1.5 \mathrm{~m}$; in seven zones no individual was registered. Individuals were found in the following micro habitats: 17 were found on aquatic vegetation, 11 in sand combined with aquatic vegetation, 9 in rock combined with aquatic vegetation, 5 in mud combined with aquatic vegetation, 2 in rock and 1 in sand. At the same time, analyzes of different water quality parameters were performed in each study area, such as: temperature, $\mathrm{pH}$, alkalinity, hardness and oxygen. 


\section{Introducción}

Las ranas del género Telmatobius están distribuidas desde el norte de los Andes ecuatorianos hasta el sur de los Andes chilenos y argentinos con un total de 63 especies; la mayoría son acuáticos y habitan en arroyos y lagos entre los 1000 a $5200 \mathrm{~m}$ de altitud (Vellard 1951), en el Perú se han reportado 27 especies en una amplia variedad de hábitats que incluyen los valles secos de la vertiente de los Andes del Pacifico, los bosques secos alto andinos y los bosques húmedos de la vertiente amazónica (Barrionuevo 2016, Ttito et al. 2016).

Telmatobius culeus es una especie endémica del altiplano peruano-boliviano, de distribución restringida al lago Titicaca y lagunas adyacentes, a elevaciones cercanas a los $3810 \mathrm{~m}$. En Bolivia se encuentra únicamente en el lago Titicaca, a diferencia de Perú, donde la especie se encuentra también en la laguna Saracocha, laguna Umayo, laguna Chajchora, laguna Arapa y río Ilave (Frost 2018, De la Riva 2005). Actualmente categorizada en el "Apéndice I" por la CITES y en "Peligro Crítico" por Unión Mundial para la Conservación de la Naturaleza (UICN). En el Perú también es considerada amenazada (DS $\mathrm{N}^{\circ}$ 004-2014-MINAGRI), debido a que enfrenta diversas amenazas como: La contaminación del lago, la colecta ilegal para la preparación de extractos con fines medicinales, la presencia de especies exóticas invasoras (trucha y pejerrey), entre las principales.

Telmatobius culeus presenta numerosos pliegues debido a una vida estrictamente acuática, pasan la mayor parte del tiempo desplazándose sobre el fondo o permanecen escondidos en la vegetación, es de cuerpo grande llegando a medir hasta $145 \mathrm{~mm}$ de SVL [snout-vent length ] (Vellard 1951) aunque se han encontrado individuos de mayor tamaño (Enrique Ramos, observación personal). El aumento de pliegues en la piel ha sido interpretado como un mecanismo compensatorio para incrementar la capacidad respiratoria de la piel en aguas con baja tensión de oxígeno (Parker 1940, Vellard 1951). Ya que en el caso de anfibios estrictamente acuáticos, las condiciones ambientales del cuerpo de agua repercuten en su fisiología y morfología e influyen directamente en la elección de algún tipo de microhábitat en especial (De Macedo 1950, Vellard 1951).

Telmatobius culeus se alimenta principalmente de peces e invertebrados acuáticos y aunque no se le reportan depredadores, se han observado gaviotas sumergiéndose en el lago para cazarlas (Jhazel Quispe, observación personal).

En el 2001 BTA-Perú hizo un estudio realizando buceo en transectos de $100 \mathrm{~m}$ x 3 m en época de menor precipitación pluvial, en la que reporto la presencia de $T$. culeus preferentemente en zonas de substrato de rocas y piedras, con una densidad de 3.33 individuos por 300 $\mathrm{m}^{2}$ para la zona de Llachon. En cambio, Genova (2011), encontró que en el sector boliviano, las ranas adultas prefieren zonas fangosas o arenosas con lugares rocosos y que los juveniles prefieren lugares totalmente rocosos que proporcionan refugio de los depredadores; por otro lado, Pérez (1998) encontro mayor número de individuos en substrato rocosos que en la vegetación, lo cual lo asocio a la preferencia en su dieta, que es mayor en crustáceos, los cuales generalmente se encuentran fijos en rocas. Flores (2013) registro 109 individuos en 110 transectos en lago menor boliviano, lo que equivale a una densidad de 1.65 individuos por $100 \mathrm{~m}^{2}$, encontrando la mayor abundancia en fondos con cobertura rocosa, disminuyendo en coberturas donde predomina la vegetación, siendo aún menor cuando el tipo de cobertura era arenoso. Callapa (2017) registró un promedio total de $0.023 \mathrm{ind} / \mathrm{m}^{2}$ que representa a tres localidades, teniendo en conocimiento que las localidades tenían una gran variación en el promedio anual de sus poblaciones.

En el presente trabajo se determinaron la abundancia relativa de Telmatobius culeus en la zona litoral lago Titicaca (sector peruano). Además se anotan observaciones sobre uso de microhábitat

\section{Material y métodos:}

Área de estudio.- El estudio se llevó a cabo en la zona litoral del lago Titicaca (sector peruano) entre los meses de junio y agosto la que se considera época seca, en donde se evaluaron 13 zonas, distribuidos según estudios previos y considerando su representatividad para el lago (Fig. 1, Tabla 1).

Se utilizó el registro de encuentros visuales (Manzanilla \& Péfaur 2000) mediante buceo con esnórquel en transectos de línea recta de 100 m considerando un ancho de banda de $2 \mathrm{~m}$ para el registro de individuos con la participación de dos buzos (investigadores) observando desde la superficie, uno a cada lado del transecto, se instalaron y se evaluaron tres transectos posicionados desde la orilla de inicio a cero metros de profundidad con dirección al centro del lago, ubicadas en paralelo y con 50 metros de separación entre ellas para cada uno de las 13 zonas de evaluación, a excepción de Z-3 (Perka Norte), todos los transectos se realizaron entre las 10:21 y 15:43h haciendo un total de 38 transectos.

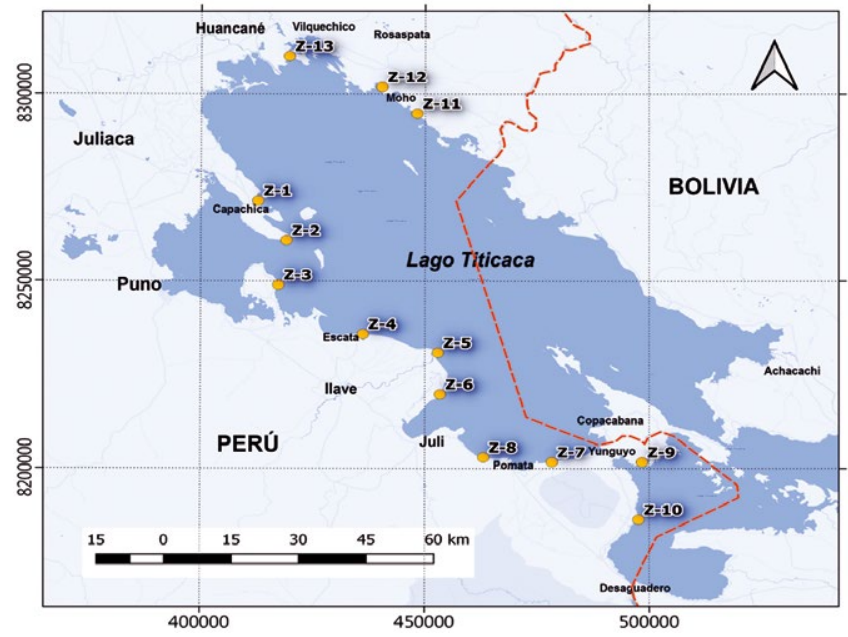

Figura 1. Ámbito de estudio y zonas de evaluación de Telmatobius culeus en el lago Titicaca (en círculos) lado peruano. 
Al encontrar un individuo se capturo temporalmente en bolsas de ziploc liberándolos al finalizar el estudio a la vez se procedió al registro de datos como: Distancia del transecto (DT), distancia perpendicular al animal (DPA), hora de inicio y hora final.

La estimación de la abundancia relativa se dio mediante el registro del tiempo utilizado (horas) en cada muestreo y el número de individuos registrado (Angulo et al. 2006), el análisis se hizo dividiendo el total de individuos registrados (total y cada zona) entre el número de horas utilizado por 02 investigadores (esfuerzo de muestreo). Se calculó el promedio de individuos en una unidad de muestreo $(100 \mathrm{~m} /$ transecto $)$ para el total de la zona litoral y cada zona de evaluación.

En cuanto a la caracterización de microhábitat, el estudio mediante buceo con esnórquel permitió visualizar el tipo de sustrato de las zonas evaluadas, se consideró el sustrato en el que se encontraron los individuos (roca, arena, vegetación acuática y fango), en casos donde el sustrato no era uniforme se combinó, dando la misma oportunidad para cada sustrato para definir el microhábitat, a la vez se registró datos como: distancia del transecto y la profundidad en el lugar exacto en donde encontramos a los individuos.

Para los análisis de los parámetros de calidad de agua en cada zona de estudio se usó la metodología de la Globlal Water Wacth (GWW) a través de un laboratorio portátil fabricado por la compañía LaMotte@ y validado por la Agencia de Protección Ambiental de los Estados Unidos de América (US EPA). se hizo la toma de muestra entre las 10:00 -15:00 horas y se evaluaron cinco parámetros, temperatura $\left(\mathrm{T}^{\circ}\right)$, oxígeno disuelto (O.D.), dureza, alcalinidad y $\mathrm{pH}$.

\section{Resultados}

De las 13 zonas evaluadas, solo en seis zonas se observó la presencia de Telmatobius culeus, donde se encontró al menos un individuo, representando esto el $46.15 \%$ del total de área evaluada y en el $53.85 \%$ restante no hubo registro alguno.

Se realizó un registro total de 45 individuos distribuidos en 17 transectos, dentro de estos transectos, en Perka Norte se limitó a evaluar solo 02 transectos, por el riesgo a hipotermia (bajas temperaturas del lago) debido al alto esfuerzo de evaluación, y donde se registró el mayor número de individuos (26) representando este el $57.8 \%$ del total de individuos, Z-9 (Ollaraya) y Z-12 (Umuchi) fueron las zonas donde se registraron el número mínimo de individuos (2) representando el $4.4 \%$ de presencia del total de individuos para cada zona de evaluación.

Se obtuvo un promedio total de 1.18 individuos para Telmatobius culeus por unidad de muestreo $(100 \mathrm{~m} /$ transecto), así mismo la abundancia relativa total registrada para toda la zona litoral fue de 2.85 ind/hora/02 personas; en Z-3 (Perka Norte) se obtuvo un promedio de 13 ind $/ 100 \mathrm{~m} /$ transecto y una abundancia relativa de 10.33 ind/hora/02 personas, las demás zonas de evaluación que registraron presencia de T. culeus, obtuvieron abundancias mucho menores (Z-1, 3.82 - Z-12, 1.6), de la misma manera el promedio de individuos por transecto se obtuvo entre 2.33 - 0.67 ind $/ 100 \mathrm{~m} /$ transecto entre $\mathrm{Z}-1 \mathrm{y}$ Z-12 respectivamente (Fig. 2).

Se realizó un esfuerzo total de $15 \mathrm{~h}$ y $48 \mathrm{~min}$ de buceo por 02 personas, cada transecto demoró 25 min en promedio, siendo el esfuerzo menor en aquellas zonas donde no se encontró ningún individuo, cada transecto tomó 14 minutos como mínimo y 87 minutos como máximo de evaluación.

Tabla 1. Información sobre frecuencia y transectos evaluados (38 en total) en el estudio de Telmatobius culeus del lago Titicaca (sector peruano). Se destaca las coordenadas de las zonas de evaluación y el número de individuos encontrados en cada zona.

\begin{tabular}{|c|c|c|c|c|c|c|}
\hline \multicolumn{2}{|c|}{ Zonas de evaluación } & \multicolumn{2}{|c|}{ Coordenadas } & \multirow{2}{*}{$\begin{array}{c}\mathbf{N}^{\circ} \text { de } \\
\text { Transectos }\end{array}$} & \multirow{2}{*}{$\mathrm{N}^{\circ}$ de individuos } & \multirow{2}{*}{ Frecuencia \% } \\
\hline Punto $\mathrm{N}^{\circ}$ & Localidad & Latitud & Longitud & & & \\
\hline Z-1 & Chifron & $15^{\circ} 38^{\prime} 0.99^{\prime \prime S}$ & $69^{\circ} 48^{\prime} 50.61^{\prime \prime} \mathrm{W}$ & 3 & 7 & 15.5 \\
\hline $\mathrm{Z}-2$ & Llachon & $15^{\circ} 43^{\prime} 45.84^{\prime \prime S}$ & $69^{\circ} 45^{\prime} 19.13^{\prime \prime} \mathrm{W}$ & 3 & 5 & 11.1 \\
\hline Z-3 & Perca norte & $15^{\circ} 50^{\prime} 11.78^{\prime \prime S}$ & $69^{\circ} 46^{\prime} 20.87^{\prime \prime} \mathrm{W}$ & 2 & 26 & 57.8 \\
\hline Z-4 & Yanaque & $15^{\circ} 57^{\prime} 21.35^{\prime \prime S}$ & $69^{\circ} 35^{\prime} 47.08^{\prime \prime W}$ & 3 & 0 & 0 \\
\hline Z-5 & Quelata & $16^{\circ} 0^{\prime} 4.32^{\prime \prime S}$ & $69^{\circ} 26^{\prime} 25.43^{\prime \prime} \mathrm{W}$ & 3 & 0 & 0 \\
\hline Z-6 & Cachipucara & $16^{\circ} 6^{\prime} 5.46^{\prime \prime S}$ & $69^{\circ} 26^{\prime} 10.07^{\prime \prime} \mathrm{W}$ & 3 & 3 & 6.7 \\
\hline$Z-7$ & Cuturapi & $16^{\circ} 15^{\prime} 54.51^{\prime \prime S}$ & $69^{\circ} 12^{\prime} 10.18^{\prime \prime} \mathrm{W}$ & 3 & 0 & 0 \\
\hline $\mathrm{Z}-8$ & Chatuma & $16^{\circ} 15^{\prime} 13.20^{\prime \prime S}$ & $69^{\circ} 20^{\prime} 45.22^{\prime \prime} \mathrm{W}$ & 3 & 0 & 0 \\
\hline Z-9 & Ollaraya & $16^{\circ} 15^{\prime} 54.53^{\prime \prime} \mathrm{S}$ & $69^{\circ} 0^{\prime} 54.62^{\prime \prime} \mathrm{W}$ & 3 & 2 & 4.4 \\
\hline Z-10 & Copani & $16^{\circ} 24^{\prime} 13.18^{\prime \prime S}$ & $69^{\circ} 1 ' 21.90 " \mathrm{~W}$ & 3 & 0 & 0 \\
\hline $\mathrm{Z}-11$ & Conima & $15^{\circ} 25^{\prime} 24.60^{\prime \prime S}$ & $69^{\circ} 28^{\prime} 57.49^{\prime \prime} \mathrm{W}$ & 3 & 0 & 0 \\
\hline $\mathrm{Z}-12$ & Umuchi & $15^{\circ} 21^{\prime} 31.61^{\prime \prime S}$ & $69^{\circ} 33^{\prime} 19.85^{\prime \prime} \mathrm{W}$ & 3 & 2 & 4.4 \\
\hline Z-13 & Koasia & $15^{\circ} 17^{\prime} 2.78^{\prime \prime S}$ & $69^{\circ} 44^{\prime} 53.89^{\prime \prime} w$ & 3 & 0 & 0 \\
\hline TOTAL & 13 & & & 38 & 45 & \\
\hline
\end{tabular}


El tipo de sustrato presento variaciones de acuerdo a la zona y a la profundidad donde realizamos la evaluación, el fango presento una capa superficial negruzca y olor sulfuroso, este combinado con vegetación acuática no permitió una visibilidad óptima en cambio las zonas donde predomino la arena se tuvo buena visibilidad presentándose en algunos casos presencia de vegetación acuática al momento de que la profundidad era mayor. Se observó la presencia de rocas medianas y pequeñas, en algunos casos combinadas con vegetación acuática donde la visibilidad en el fondo fue buena, la vegetación acuática estuvo compuesta principalmente por Schoenoplectus tatora y Myriophyllum sp. De los 45 individuos encontrados, 17 fueron encontrados sobre vegetación acuática, 11 en arena combinado con vegetación acuática, 9 en roca combinado con vegetación acuática, 5 en fango combinado con vegetación acuática, 2 en roca y 1 en arena. Por lo que se puede indicar que T. culeus uso preferentemente el microhábitat vegetación acuática y rocoso con vegetación acuática (Fig. 3).

Para los análisis de calidad de agua, el máximo valor de oxígeno disuelto y temperatura se registró en la zona de Perka Norte mientras que los mínimos en la zona de Copani. Los valores del pH en las trece zonas se registraron dentro de lo establecido por los Estándares de Calidad Ambiental, Categoría 4: para la conservación del ambiente acuático (ECA Categoría 4: 6,5 a 9,0) [DS $\mathrm{N}^{\circ}$ 004-2017-MINAM]. Según el Índice de Dureza del Agua, 09/13 zonas de estudio presentaron aguas "muy duras"; las zonas de Umuchi y Koasia, respectivamente presentaron aguas "duras" y la zona de Quelata aguas "media". En el caso de la alcalinidad, la zona de Copani presentó un valor bajo, mientras que las demás presentan valores entre medio y alto (Tabla 2).

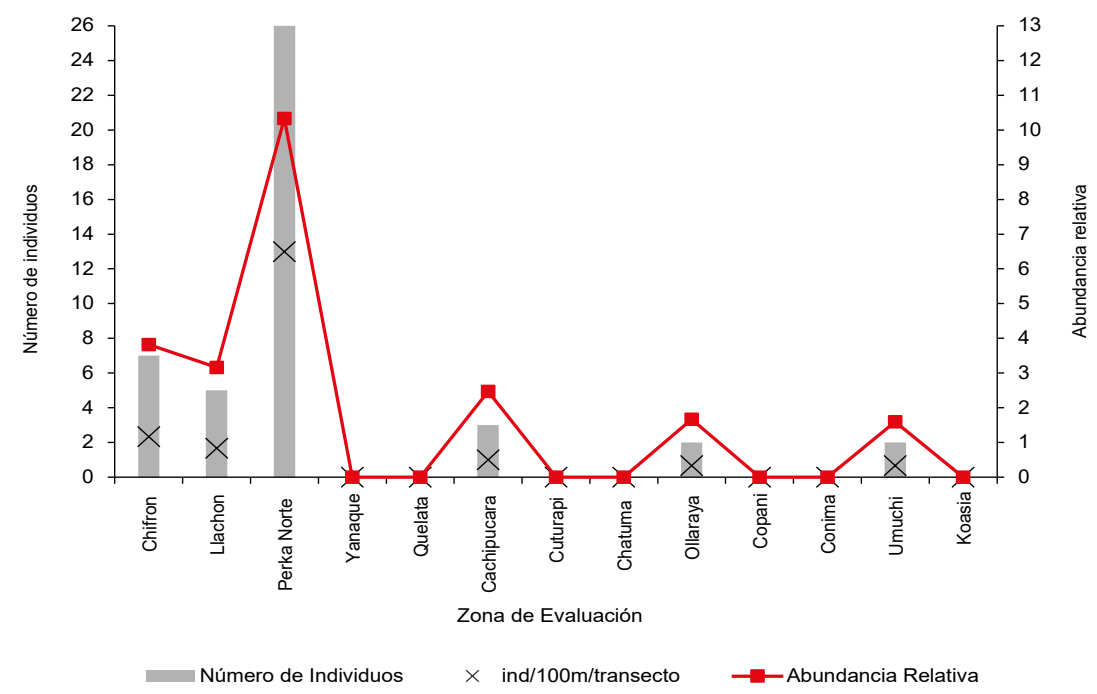

Figura 2. Número de individuos, número de individuos promedio por unidad de evaluación (100m/transecto) y Abundancia relativa por cada zona de evaluación de Telmatobius culeus en el lago Titicaca (sector peruano).

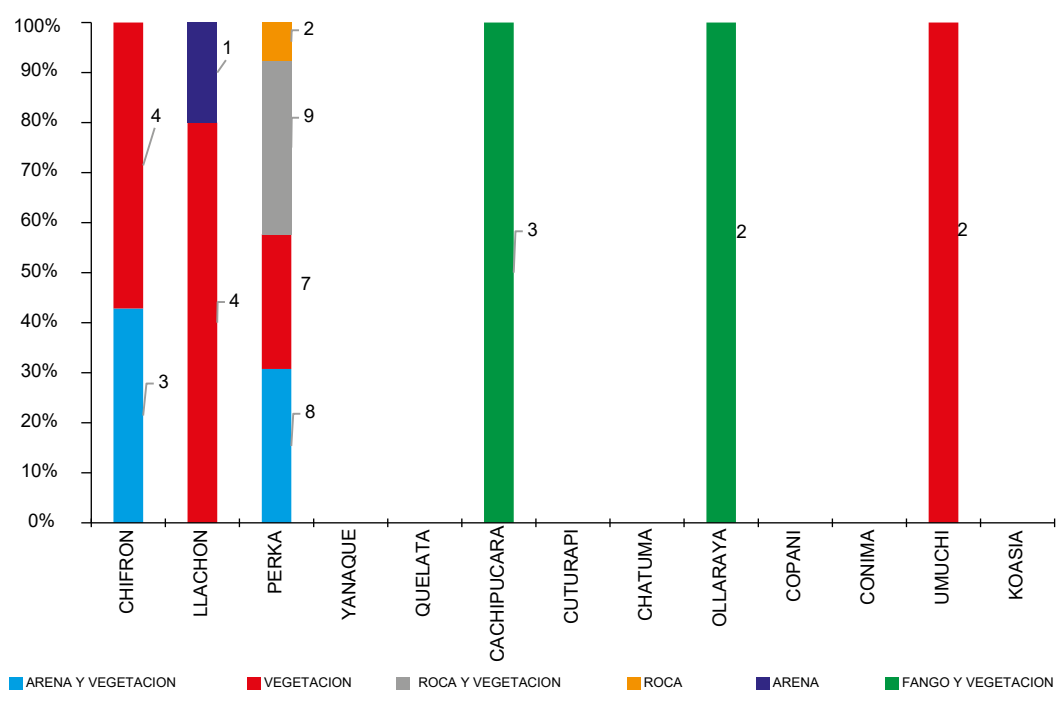

Figura 3. Se muestra el porcentaje de microhábitat por zona de evaluación de Telmatobius culeus en el lago Titicaca (sector peruano). Los numeros indican el número de individuos en cada microhabitat. 
Tabla 2. Información sobre calidad de agua en cada zona de evaluación de Telmatobius culeus en el lago Titicaca (sector peruano).

\begin{tabular}{|c|c|c|c|c|c|c|c|c|}
\hline $\begin{array}{l}\text { Zonas de } \\
\text { evaluación }\end{array}$ & Fecha & $\begin{array}{l}\text { Hora de } \\
\text { muestreo }\end{array}$ & $\begin{array}{l}\text { Temperatura } \\
\text { agua }{ }^{\circ} \mathrm{C}\end{array}$ & $\mathrm{pH}$ & Alcalinidad mg/L & Dureza mg/L & $\begin{array}{l}\text { Oxígeno disuelto } \\
(\mathrm{mg} / \mathrm{L})\end{array}$ & $\begin{array}{l}\text { Porcentaje saturación } \\
\text { de oxígeno }(\%)\end{array}$ \\
\hline Chifron & $11 / 06 / 2017$ & $12: 46$ & 13 & 8 & 110 & 340 & 6.7 & 61 \\
\hline Llachon & $17 / 06 / 2017$ & $12: 05$ & 15.5 & 8 & 135 & 360 & 5.9 & 57 \\
\hline Perka norte & $24 / 06 / 2017$ & $11: 30$ & 16 & 8.5 & 115 & 320 & 8 & 81 \\
\hline Yanaque & $8 / 07 / 2017$ & $10: 50$ & 13.5 & 8 & 105 & 320 & 6.1 & 58 \\
\hline Quelata & 9/07/2017 & $11: 55$ & 13 & 6.5 & 125 & 130 & 6.3 & 59 \\
\hline Cachipucara & $9 / 07 / 2017$ & $14: 45$ & 14 & 7.5 & 135 & 310 & 6 & 59 \\
\hline Cuturapi & 29/07/2017 & $13: 11$ & 15.8 & 7 & 100 & 300 & 6.1 & 60 \\
\hline Chatuma & $30 / 07 / 2017$ & $11: 30$ & 14.8 & 7.5 & 105 & 320 & 5.8 & 57 \\
\hline Ollaraya & $5 / 08 / 2017$ & $13: 26$ & 14.8 & 8 & 80 & 360 & 6 & 58 \\
\hline Copani & $6 / 08 / 2017$ & $10: 30$ & 12.9 & 7.5 & 70 & 340 & 4.4 & 40 \\
\hline Conima & 19/08/2017 & $11: 20$ & 13 & 7.5 & 95 & 340 & 6.2 & 58 \\
\hline Umuchi & 20/08/2017 & $11: 10$ & 12.8 & 8 & 100 & 280 & 6.3 & 58 \\
\hline Koasia & $27 / 08 / 2017$ & $14: 13$ & 14 & 7.5 & 100 & 230 & 6.5 & 63 \\
\hline
\end{tabular}

\section{Discusión}

Según lo registrado de acuerdo a la metodología usada, existe poca presencia de T. culeus en $100 \mathrm{~m}$ de la zona litoral del lago Titicaca, a excepción de la zona de Perka Norte done se registró un alto número de individuos. A diferencia de lo encontrado por BTA-Perú (2002) que registró el más alto número de individuos en la localidad de Llachón con 10 individuos siendo esta zona la de mayor densidad con 3.33 individuos por $300 \mathrm{~m}^{2}$, esto puede ser debido a que en su metodología utilizaron buceo con tanque en transectos de $300 \mathrm{~m}^{2}$, lo cual le permite mayor cobertura de avistamiento a partir de dos metros de profundidad. Flores (2013) realizo un estudio mediante buceo en el lado boliviano usando transectos de $30 \times 2 \mathrm{~m}$ a una distancia máximo de $35 \mathrm{~m}$ de la orilla en la que registro un total de 109 individuos en 110 transectos, obteniendo una densidad de 1.65 individuos por cada $100 \mathrm{~m}^{2}$

En base a la caracterización realizada, en nuestro estudio se puede indicar que el sustrato donde se registró el mayor número de individuos fue el de vegetación acuática, siendo 17 el total de individuos encontrados, sin embargo, BTA-Perú (2002) reportó la presencia preferentemente en zonas de rocas y piedras, sin preferencia por algún tipo de vegetación. Flores (2013), en el caso de la cobertura de fondo menciona que existe una mayor abundancia en fondos con cobertura rocosa, al contrario, se puede observar una menor abundancia en coberturas donde predomina la vegetación, siendo aún menor cuando el tipo de cobertura es arenoso ya que de los 109 individuos encontrados en su estudio, 53 fueron registrados sobre rocas, 44 sobre vegetación y 12 en arena. Es posible inferir que las ranas prefieren las superficies rocosas y evitan las zonas con cobertura vegetal. La explicación de que no exista una dependencia entre el tipo de vegetación podría deberse a la presencia de especies de anfípodos, gasterópodos y peces que podrían estar presentes en superficies rocosas (Pérez 1998). en temporadas lluviosas, también menciona que los componentes de calidad de agua que influyen en las densidades de T. culeus son la alcalinidad, amoniaco y dureza del agua, los valores de dureza del agua en el lago Titicaca son altos (duras) por encima de $150 \mathrm{mg} / \mathrm{L} \mathrm{CaCO} 3$ y T. culeus muestra adaptaciones particulares, ya que a mayor dureza del agua existe una mayor densidad en sus poblaciones. Sin embargo, en nuestro estudio en la localidad donde se registró la mayor abundancia de individuos estuvo determinado por el oxígeno siendo la zona de Perka Norte en la que se encontró un porcentaje de saturación de oxígeno "adecuado" con $81 \%$.

\section{Literatura citada}

Angulo A., J. V. Rueda-Almonacid, J. V. Rodríguez-Mahecha \& E. La Mar ca (Eds). 2006. Técnicas de inventario y monitoreo para los anfibios de la región tropical andina. Conservación Internacional. Serie Manuales de Campo № 2. Panamericana Formas e Impresos S.A., Bogotá D.C. $298 \mathrm{pp}$

Barrionuevo J.S. 2016. Frogs at the summits: phylogeny of the Andean frogs of the genus Telmatobius (Anura, Telmatobiidae) based on phenotypic characters. Cladistics 0 (2016): 1- 18pp. https://doi.org/10.1111/cla.12158

Biotecnología Agropecuaria Perú. 2002. Evaluación de la población de rana gigante del lago, Telmatobius culeus. Evaluación poblacional. Informe final segunda evaluación poblacional. Vol II. ALT-PNUD 125 pp.

Callapa G. 2017. Estado poblacional de Telmatobius culeus (anura: Telmatobiidae) en tres localidades en la zona boliviana del lago Titicaca. La Paz, Bolivia. Universidad Mayor de San Simón. Cochabamba, Bolivia 66 pp.

De la Riva I. 2005. Bolivian frogs of the genus Telmatobius: sinopsis, taxonomic, comments, and description of a new species. Monografías de herpetología. 7. 65-101 pp.

De Macedo H. 1950. Anotaciones para el conocimiento zoológico del género Batrachophrynus (Amphibia: Salientia). Tesis universitaria para optar el título de Bachiller.

Callapa (2017), registro densidades altas de T. culeus 
Lima. Universidad Nacional Mayor de San Marcos. 27 Pp. Acceso: 11/01/16

DS N 004-2017-MINAM.2017. Aprueban Estándares de Calidad Ambiental (ECA) para Agua y establecen Disposiciones Complementarias. Normas Legales, El Peruano. Miércoles 7 de junio de 2017:10-19

Flores V. 2013. Preferencia de hábitat y densidad de Telmatobius culeus (Familia: Ceratophryidae) en el Lago Titicaca. Tesis de Licenciatura. UMSA. La Paz, Bolivia 59 pp.

Frost, Darrel R. 2018. Amphibian Species of the World: an Online Reference. Version 6.0 (Date of access). Electronic Database accessible at http://research.amnh.org/herpetology/amphibia/index.html. American Museum of Natural History, New York, USA.

Genova M.I. 2011. Density and habitat preferences of Lake Titicaca frog (Telmatobius culeus) at NorthWest of Copacabana peninsula. Master Thesis. WageningenUniversiteit. $73 \mathrm{pp}$.

Manzanilla, J. \& J. Péfaur. 2000. Consideraciones sobre métodos y técnicas de campo para el estudio de anfibios y reptiles. Ecol. Lat. Am. 7 (1-2): 17-30

Ministerio de Agricultura y Riego (MINAGRI). 2014. Decreto Supremo que aprueba la actualización de la lista de clasificación y categorización de las especies amenazadas de fauna silvestre legalmente protegidas. Decreto Supremo 004-2014-MINAGRI. El Peruano, Lima Perú, abril 08. Normas legales: 520497-520504.

Parker H. W. 1940. Percy SladenTrust Expedition to lake Titi- caca under the leadership of Me. Cary Gilson, M. A. XII Amphibia. Transactions of the Linnean Society of London 3 (1): 203-216.

Pérez E. 1998. Dieta y ciclo gametogénico anual de Telmatobius culeus en el Lago Titicaca. Tesis para optar el Titulo de Licenciada en Biología. La Paz: Universidad Mayor de San Andrés. (Biblioteca del Instituto de Ecología).

Ttito A., C.Z. Landauro \& P.J. Venegas, et al. 2016. A new species of Telmatobius Wiegmann, 1834, from the eastern Cordillera Central of the Andes, Perú (Anura: Telmatobiidae), with description of its tadpole, and range extension of T. mendelsoni De la Riva et al., 2012. Ann Carnegie Mus 83: 255-268 pp.

Vellard J. 1951. Estudio sobre batracios andinos I. el grupo Telmatobius sp. y formas afines memorias del Museo de Historia Natural. Javier Prado, Lima-Perú.
Agradecimientos:

Nuestro agradecimiento a Gariz Riveros Urbina y Kewin Otazu Mamani por su participación en el trabajo de campo durante la investigación.

Conflicto de intereses:

Los autores no incurren en conflictos de intereses.

Rol de los autores:

VERR Concepción de la investigación, muestreos y redacción del artículo. JAQC muestreos y redacción del artículo. RKEP muestreos y redacción del artículo. VERR, JAQC, RKEP revisaron y aprobaron el manuscrito.

Fuentes de financiamiento:

El presente trabajo se realizó gracias al financiamiento de Denver Zooological Foundation.

Aspectos éticos / legales:

Servicio Nacional Forestal y de Fauna Silvestre (SERFOR) del Ministerio de Agricultura y Riego, Permiso de Investigación Científica $\mathrm{N}^{\circ}$ 288-2016-SERFOR-DGGSPFFS. 